\title{
Analysis of Transient Dynamic Behaviour of Spherical Cavity in Viscoelastic Soil Medium
}

\author{
J. P. Dwivedi, V. P. Singh, and Radha Krishna Lal \\ Department of Mechanical Engineering, Indian Institute of Technology, Banaras Hindu University, Varanasi 221005, India \\ Correspondence should be addressed to Radha Krishna Lal; radhakrishna773@gmail.com
}

Received 30 October 2012; Accepted 27 November 2012

Academic Editors: Y.-H. Lin and W. O. Wong

Copyright (C) 2013 J. P. Dwivedi et al. This is an open access article distributed under the Creative Commons Attribution License, which permits unrestricted use, distribution, and reproduction in any medium, provided the original work is properly cited.

Stress, displacement, and pore pressure of a partially sealed spherical cavity in viscoelastic soil condition have been obtained in Laplace transform domain. Solutions of axisymmetric surface load and fluid pressure are derived.

\section{Introduction}

Biot $[1,2]$ presented the propagation theory of elastic waves and the general solutions for fluid-saturated porous viscoelastic medium. Akkas and Zakout [3] discussed the solution for the transient response for an axisymmetric and nontorsional load of an infinite, isotropic, elastic medium containing a spherical cavity with and without thin elastic shell embedment. In this paper, considering a viscoelastic model presented by Eringen [4], the transient response of a spherical cavity with a partially sealed shell embedded in viscoelastic soil is investigated. The solutions of stresses, displacements and pore pressure induced by axisymmetric surface load and fluid pressure are derived in Laplace transform domain. Durbin's [5] inverse Laplace transform is used to analyze the influence of partial permeable property of boundary and relative rigidity of shell and soil on the transient response of the spherical cavity. The solutions of permeable and impermeable boundary without shell are considered as two extreme cases.

\section{Basic Equations and Solutions}

In infinite viscoelastic saturated soil, a thin elastic shell shown in Figure 1 with inner radius $a$, outer radius $b$, and thickness $h=b-a$, has been bored. $(r, \theta, \phi)$ are the spherical coordinates, where $\theta$ and $\phi$ are the meridional and circumferential angles, respectively, $\sigma_{r}, \sigma_{\theta}, \sigma_{\phi}$ are nonvanishing components of stress tensor in case of an axisymmetric nontorsional load, that is, independent of $\theta$ and $\phi$ acting on the shell surface.

In spherical coordinate system $(r, \theta, \phi)$, the equilibrium equation for soil mass is

$$
\frac{\partial \sigma_{r}}{\partial r}+\frac{2 \sigma_{r}-\sigma_{\theta}-\sigma_{\phi}}{r}=\frac{\partial^{2}}{\partial t^{2}}\left(\rho u_{r}+\rho_{f} w_{r}\right),
$$

where $u_{r}$ and $w_{r}$ are radial displacement of soil skelton and displacement of pore fluid with respect to soil skelton, respectively; $\rho=(1-n) \rho_{s}+\eta \rho_{f}$, the density of soil; $\rho_{f}$ and $\rho_{s}$ are densities of fluid and soil grains respectively; $n$ is porosity.

The pore fluid equilibrium equation is given by

$$
-\frac{\partial p}{\partial r}=\frac{\partial^{2}}{\partial t^{2}}\left(\rho_{f} u_{r}+\frac{\rho_{f}}{n} w_{r}\right)+\frac{\eta_{0}}{k_{d}} \frac{\partial w_{r}}{\partial t},
$$

where $p$ is excess pore pressure; $\eta_{0}$ is the fluid viscosity, and $k_{d}$ is the intrinsic permeability of soil.

Soil is not an ideal medium. Due to overcoming the interior friction of soil, a part of energy of the propagation wave is changed into heat energy during the propagation. This 


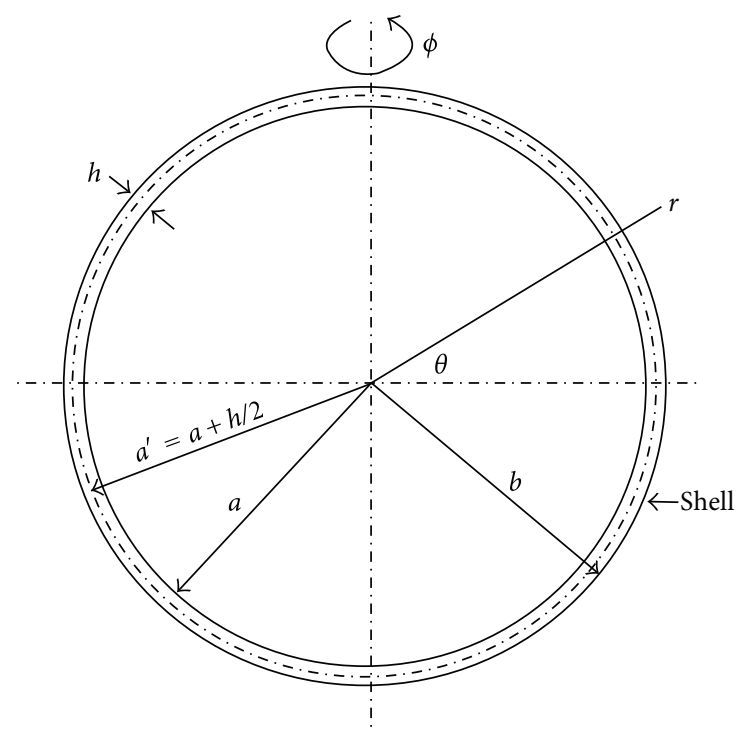

FIGURE 1: Geometry of the problem.

property is known as damping of material. Assuming that the viscoelastic property of soil may be simulated by Kelvin-Voigt model. Following Eringen [4], the stress-strain relationship is expressed as

$$
\begin{gathered}
\sigma_{r}=\lambda e+2 G \frac{\partial u_{r}}{\partial r}+\lambda^{\prime} \frac{\partial e}{\partial t}+2 G^{\prime} \frac{\partial}{\partial t}\left(\frac{\partial u_{r}}{\partial r}\right)-\alpha p \\
\sigma_{\theta}=\sigma_{\phi}=\lambda e+2 G \frac{u_{r}}{r}+\lambda^{\prime} \frac{\partial e}{\partial t}+2 G^{\prime} \frac{\partial}{\partial t}\left(\frac{u_{r}}{r}\right)-\alpha p \\
p=M \xi-\alpha M e
\end{gathered}
$$

where $e=\partial u_{r} / \partial r+2 u_{r} / r$ and $\xi=-\left(\partial w_{r} / \partial r+2 w_{r} / r\right)$, dilations of solid and fluid, respectively; $\lambda$ and $G$ are Lame constants of the bulk material; $\lambda^{\prime}$ and $G^{\prime}$ are the dilatant and shear constant of the viscoelastic soil; $\alpha$ and $M$ are the compressibility parameters of the two phase medium, $0 \leq$ $\alpha \leq 1,0 \leq M \leq \infty$ and $M \rightarrow \infty, \alpha \rightarrow 1$ for a material with incompressible constituents.

Substituting (3), (4), and (5) into (1) and (2), the governing equations of the transient response of a spherical cavity in viscoelastic solid condition can be reduced as

$$
\begin{gathered}
\left(\lambda+2 G+\alpha^{2} M\right) \frac{\partial e}{\partial r}+\left(\lambda^{\prime}+2 G^{\prime}\right) \frac{\partial}{\partial t}\left(\frac{\partial e}{\partial r}\right)-\alpha M \frac{\partial \xi}{\partial r} \\
=\frac{\partial^{2}}{\partial t^{2}}\left(\rho u_{r}+\rho_{f} w_{r}\right), \\
\alpha M \frac{\partial e}{\partial r}-M \frac{\partial \xi}{\partial r}=\frac{\partial^{2}}{\partial t^{2}}\left(\rho_{f} u_{r}+\frac{\rho_{f}}{n} w_{r}\right)+\frac{\eta_{0}}{k_{d}} \frac{\partial w_{r}}{\partial t} .
\end{gathered}
$$

Now, displacements $u_{r}$ and $w_{r}$ are assumed to be of the forms

$$
\begin{aligned}
& u_{r}=\frac{\partial}{\partial r}\left(\frac{U(r) \cos \omega t}{r}\right), \\
& w_{r}=\frac{\partial}{\partial r}\left(\frac{W(r) \cos \omega t}{r}\right),
\end{aligned}
$$

for solving (6) and (7). Consider

$$
\begin{aligned}
& e=\frac{\partial u_{r}}{\partial r}+\frac{2 u_{r}}{r}, \\
& \frac{\partial e}{\partial r}=\frac{\partial^{2} u_{r}}{\partial r^{2}}+\frac{2}{r} \frac{\partial u_{r}}{\partial r}-\frac{2}{r^{2}} u_{r}, \\
& u_{r}=\frac{\partial}{\partial r}\left(\frac{U(r) \cos \omega t}{r}\right), \\
& u_{r}=\frac{1}{r} \frac{\partial}{\partial r}(U(r) \cos \omega t)-\frac{U(r) \cos \omega t}{r^{2}}, \\
& \frac{\partial u_{r}}{\partial r}=\frac{1}{r} \frac{\partial^{2}}{\partial r^{2}}(U(r) \cos \omega t)-\frac{2}{r^{2}} \frac{\partial}{\partial r}(U(r) \cos \omega t) \\
& \quad+\frac{2}{r^{3}} U(r) \cos \omega t, \\
& \frac{\partial^{2} u_{r}}{\partial r^{2}}=\frac{1}{r} \frac{\partial^{3}}{\partial r^{3}}(U(r) \cos \omega t)-\frac{3}{r^{2}} \frac{\partial^{2}}{\partial r^{2}}(U(r) \cos \omega t) \\
& \quad+\frac{6}{r^{3}} \frac{\partial}{\partial r}(U(r) \cos \omega t)-\frac{6}{r^{4}} U(r) \cos \omega t .
\end{aligned}
$$

Then,

$$
\begin{aligned}
\frac{\partial e}{\partial r} & =\frac{1}{r} \frac{\partial^{3}}{\partial r^{3}}(U(r) \cos \omega t)-\frac{1}{r^{2}} \frac{\partial^{2}}{\partial r^{2}}(U(r) \cos \omega t) \\
& =\frac{\partial}{\partial r}\left(\frac{1}{r} \frac{\partial^{2}}{\partial r^{2}}(U(r) \cos \omega t)\right)=\frac{\partial}{\partial r}\left(\frac{1}{r} \nabla^{2}(U(r) \cos \omega t)\right)
\end{aligned}
$$

Similarly,

$$
\begin{aligned}
\frac{\partial \xi}{\partial r} & =-\frac{\partial}{\partial r}\left(\frac{1}{r} \frac{\partial^{2}}{\partial r^{2}}(W(r) \cos \omega t)\right) \\
& =-\frac{\partial}{\partial r}\left(\frac{1}{r} \nabla^{2}(W(r) \cos \omega t)\right) .
\end{aligned}
$$

Substituting (8), (9), (11), and (12), into (6), we obtain

$$
\begin{aligned}
(\lambda+ & \left.2 G+\alpha^{2} M\right) \frac{\partial}{\partial r}\left(\frac{1}{r} \nabla^{2}(U(r) \cos \omega t)\right) \\
& +\left(\lambda^{\prime}+2 G^{\prime}\right) \frac{\partial}{\partial t}\left(\frac{\partial}{\partial r}\left(\frac{1}{r} \nabla^{2}(U(r) \cos \omega t)\right)\right) \\
& -\alpha M \frac{\partial}{\partial r}\left(\frac{1}{r} \nabla^{2}(W(r) \cos \omega t)\right)
\end{aligned}
$$




$$
\begin{gathered}
=\frac{\partial^{2}}{\partial t^{2}}\left(\rho \frac{\partial}{\partial r}\left(\frac{1}{r} \nabla^{2}(U(r) \cos \omega t)\right)\right. \\
\left.+\rho_{f} \frac{\partial}{\partial r}\left(\frac{1}{r} \nabla^{2}(W(r) \cos \omega t)\right)\right), \\
\frac{\partial}{\partial r}\left[\frac{1}{r}\left(\lambda+2 G+\alpha^{2} M\right) \nabla^{2} U(r) \cos \omega t\right. \\
\left.+\left(\lambda^{\prime}+2 G^{\prime}\right) \frac{\partial}{\partial t} \nabla^{2} U(r) \cos \omega t+\alpha M \nabla^{2} W(r) \cos \omega t\right] \\
=\frac{\partial}{\partial r}\left[\frac{1}{r} \frac{\partial^{2}}{\partial t^{2}}\left(\rho U(r) \cos \omega t+\rho_{f} W(r) \cos \omega t\right)\right] .
\end{gathered}
$$

Integrating w.r.t. $r$, we have

$$
\begin{aligned}
& \left\{\left(\lambda+2 G+\alpha^{2} M\right)+\left(\lambda^{\prime}+2 G^{\prime}\right) \frac{\partial}{\partial t}\right\} \nabla^{2} U(r) \cos \omega t \\
& \quad+\alpha M \nabla^{2} W(r) \cos \omega t \\
& =\frac{\partial^{2}}{\partial t^{2}}\left(\rho U(r) \cos \omega t+\rho_{f} W(r) \cos \omega t\right) \\
& {\left[\left(\lambda+2 G+\alpha^{2} M\right) \nabla^{2}+\left(\lambda^{\prime}+2 G^{\prime}\right) \frac{\partial}{\partial t} \nabla^{2}-\rho \frac{\partial^{2}}{\partial t^{2}}\right]} \\
& \quad \times U(r) \cos \omega t+\left(\alpha M \nabla^{2}-\rho_{f} \frac{\partial^{2}}{\partial t^{2}}\right) W(r) \cos \omega t=0
\end{aligned}
$$

where $\nabla^{2}=\partial^{2} / \partial r^{2}$.

Substituting (8) to (12), in (7), we obtain

$$
\begin{aligned}
\alpha M & \frac{\partial}{\partial r}\left(\frac{1}{r} \nabla^{2} U(r) \cos \omega t\right)+M \frac{\partial}{\partial r}\left(\frac{1}{r} \nabla^{2} W(r) \cos \omega t\right) \\
= & \frac{\partial^{2}}{\partial t^{2}}\left\{\rho_{f} \frac{\partial}{\partial r}\left(\frac{1}{r} U(r) \cos \omega t\right)+\frac{\rho_{f}}{n} \frac{\partial}{\partial r}\left(\frac{1}{r} W(r) \cos \omega t\right)\right\} \\
& +\frac{\eta_{0}}{k_{d}} \frac{\partial}{\partial t}\left(\frac{\partial}{\partial r}\left(\frac{1}{r} W(r) \cos \omega t\right)\right),
\end{aligned}
$$$$
\frac{\partial}{\partial r}\left[\frac{1}{r}\left(\alpha M \nabla^{2} U(r) \cos \omega t+M \nabla^{2} W(r) \cos \omega t\right)\right]
$$$$
=\frac{\partial}{\partial r}\left[\frac { 1 } { r } \left(\frac{\partial^{2}}{\partial t^{2}} \rho_{f} U(r) \cos \omega t+\frac{\partial^{2}}{\partial t^{2}} \frac{\rho_{f}}{n} W(r) \cos \omega t\right.\right.
$$

$$
\left.\left.+\frac{\eta_{0}}{k_{d}} \frac{\partial}{\partial t} W(r) \cos \omega t\right)\right]
$$

Integrating w.r.t. $r$, we have

$\alpha M \nabla^{2} U(r) \cos \omega t+M \nabla^{2} W(r) \cos \omega t$

$$
\begin{gathered}
=\frac{\partial^{2}}{\partial t^{2}} \rho_{f} U(r) \cos \omega t+\frac{\partial^{2}}{\partial t^{2}} \frac{\rho_{f}}{n} W(r) \cos \omega t \\
+\frac{\eta_{0}}{k_{d}} \frac{\partial}{\partial t} W(r) \cos \omega t, \\
{\left[\alpha M \nabla^{2}-\rho_{f} \frac{\partial^{2}}{\partial t^{2}}\right] U(r) \cos \omega t} \\
+\left[M \nabla^{2}-\frac{\rho_{f}}{n} \frac{\partial^{2}}{\partial t^{2}}-\frac{\eta_{0}}{k_{d}} \frac{\partial}{\partial t}\right] W(r) \cos \omega t=0 .
\end{gathered}
$$

Taking Laplace transform in (14) yields

$$
\begin{aligned}
& {\left[\left(\lambda+2 G+\alpha^{2} M\right) \nabla^{2}\left(\frac{s}{s^{2}+\omega^{2}}\right)+\left(\lambda^{\prime}+2 G^{\prime}\right) \nabla^{2}\left(\frac{-\omega^{2}}{s^{2}+\omega^{2}}\right)\right.} \\
& \left.-\rho\left(\frac{-\omega^{2} s}{s^{2}+\omega^{2}}\right)\right] U(r) \\
& +\left(\alpha M \nabla^{2}\left(\frac{s}{s^{2}+\omega^{2}}\right)-\rho_{f}\left(\frac{-\omega^{2} s}{s^{2}+\omega^{2}}\right)\right) W(r)=0, \\
& {\left[\left(\lambda+2 G+\alpha^{2} M\right) \nabla^{2}-\frac{\omega^{2}}{s}\left(\lambda^{\prime}+2 G^{\prime}\right) \nabla^{2}+\rho \omega^{2}\right] \frac{U(r) s}{s^{2}+\omega^{2}}} \\
& \quad+\left[\alpha M \nabla^{2}+\rho_{f} \omega^{2}\right] \frac{W(r) s}{s^{2}+\omega^{2}}=0 .
\end{aligned}
$$

Taking Laplace transform in (14) yields

$$
\begin{aligned}
& {\left[\alpha M \nabla^{2}\left(\frac{s}{s^{2}+\omega^{2}}\right)-\rho_{f}\left(\frac{-\omega^{2} s}{s^{2}+\omega^{2}}\right)\right] U(r)} \\
& +\left[M \nabla^{2}\left(\frac{s}{s^{2}+\omega^{2}}\right)-\frac{\rho_{f}}{n}\left(\frac{-\omega^{2} s}{s^{2}+\omega^{2}}\right)-\frac{\eta_{0}}{k_{d}}\left(\frac{-\omega^{2}}{s^{2}+\omega^{2}}\right)\right] \\
& \quad \times W(r)=0, \\
& \left(\alpha M \nabla^{2}+\omega^{2} \rho_{f}\right) \frac{U(r) s}{s^{2}+\omega^{2}}+\left[\left(M \nabla^{2}+\frac{\rho_{f}}{n} \omega^{2}\right)+\frac{\eta_{0}}{k_{d}} \frac{\omega^{2}}{s}\right] \\
& \quad \times \frac{W(r) s}{s^{2}+\omega^{2}}=0 .
\end{aligned}
$$

In a great range of vibration frequencies, viscoelastic damp coefficient of rock and soft soil may be assumed as a constant. The dimensionless damp coefficient $\eta$ is considered as

$$
\eta=\frac{\lambda^{\prime}}{\lambda}=\frac{G^{\prime}}{G}
$$


Also,

$$
\begin{gathered}
\lambda^{*}=\frac{\lambda}{G}, \quad M^{*}=\frac{M}{G}, \quad \rho^{*}=\frac{\rho_{f}}{\rho}, \\
b^{*}=\frac{\eta_{0} a^{\prime}}{k_{d} \sqrt{\rho G}}, \quad \frac{\rho}{G} \sim 1
\end{gathered}
$$

are nondimensional Lame constant, compressibility parameter, fluid density, and permeability coefficient of soil respectively. $b$ is the radius of spherical shell, $a^{\prime}=b-h / 2=a+h / 2$.

Using (19) and (20) in (17) yields

$$
\begin{aligned}
& {\left[\left(\left(\lambda^{*}+2\right)+\alpha^{2} M^{*}\right) \nabla^{2}-\frac{\omega^{2}}{s}\left(\eta \lambda^{\prime}+2 \eta\right) \nabla^{2}+\omega^{2}\right] \frac{U(r) s}{s^{2}+\omega^{2}}} \\
& \quad+\left[\alpha M^{*} \nabla^{2}+\rho^{*} \omega^{2}\right] \frac{W(r) s}{s^{2}+\omega^{2}}=0, \\
& {\left[\left\{\left(\lambda^{*}+2\right)\left(1-\frac{\omega^{2}}{s} \eta\right)+\alpha^{2} M^{*} s\right\} \nabla^{2}+\omega^{2}\right] \frac{U(r) s}{s^{2}+\omega^{2}}} \\
& \quad+\left(\alpha M^{*} \nabla^{2}+\rho^{*} \omega^{2}\right) \frac{W(r) s}{s^{2}+\omega^{2}}=0 .
\end{aligned}
$$

Similarly, using (19) and (20) in (18) yields

$$
\begin{aligned}
& \left(\alpha M^{*} \nabla^{2}+\rho^{*} \omega^{2}\right) \frac{U(r) s}{s^{2}+\omega^{2}} \\
& +\left[\left(M^{*} \nabla^{2}+\frac{\rho^{*} \omega^{2}}{n}\right)+b^{*} \frac{\omega^{2}}{s}\right] \frac{W(r) s}{s^{2}+\omega^{2}}=0, \\
& \left(\alpha M^{*} \nabla^{2}+\rho^{*} \omega^{2}\right) \frac{U(r) s}{s^{2}+\omega^{2}} \\
& +\left[M^{*} \nabla^{2}+\left(\frac{\rho^{*}}{n}+\frac{b^{*}}{s}\right) \omega^{2}\right] \frac{W(r) s}{s^{2}+\omega^{2}}=0 .
\end{aligned}
$$

Solving (21) and (22) yields

$$
\begin{aligned}
& {\left[\left\{\left(\left(\lambda^{*}+2\right)\left(1-\frac{\omega^{2}}{s} \eta\right)+\alpha^{2} M^{*}\right) \nabla^{2}+\omega^{2}\right\}\right.} \\
& \left.\quad \times\left\{M^{*} \nabla^{2}+\left(\frac{\rho^{*}}{n}+\frac{b^{*}}{s}\right) \omega^{2}\right\}-\left\{\left(\alpha M^{*} \nabla^{2}+\rho^{*} \omega^{2}\right)\right\}^{2}\right] \\
& \quad \times\left[\frac{U(r) s}{s^{2}+\omega^{2}}, \frac{W(r) s}{s^{2}+\omega^{2}}\right]=0,
\end{aligned}
$$

$$
\begin{aligned}
& {\left[\left(\lambda^{*}+2\right)\left(1-\frac{\omega^{2}}{s} \eta\right) M^{*} \nabla^{4}\right.} \\
& +\left\{\left(\left(\lambda^{*}+2\right)\left(1-\frac{\omega^{2}}{s} \eta\right)+\alpha^{2} M^{*}\right)\left(\frac{\rho^{*}}{n}+\frac{b^{*}}{s}\right)\right. \\
& \left.\quad+M^{*}\left(1-2 \alpha \rho^{*}\right)\right\} \omega^{2} \nabla^{2} \\
& \left.+\left\{\left(\frac{\rho^{*}}{n}+\frac{b^{*}}{s}\right)-\left(\rho^{*}\right)^{2}\right\} \omega^{4}\right](U, W)\left(\frac{s}{s^{2}+\omega^{2}}\right)=0 .
\end{aligned}
$$

Equation (24) can be written as

$$
\left(\nabla^{2}-\gamma_{1}^{2}\right)\left(\nabla^{2}-\gamma_{2}^{2}\right)(U, W)\left(\frac{s}{s^{2}+\omega^{2}}\right)=0,
$$

where $\gamma_{1}$ and $\gamma_{2}$ are the complex wave number of two dilation waves, that is,

$$
\begin{aligned}
& \gamma_{1}^{2}=\frac{\alpha_{1}-\sqrt{\alpha_{1}-4 \alpha_{2}}}{2}, \\
& \gamma_{2}^{2}=\frac{\alpha_{1}+\sqrt{\alpha_{1}-4 \alpha_{2}}}{2},
\end{aligned}
$$

with

$$
\begin{aligned}
\alpha_{1}= & {\left[\left(\left(\lambda^{*}+2\right)\left(1-\left(\omega^{2} / s\right) \eta\right)+\alpha^{2} M^{*}\right)\right.} \\
& \left.\times\left(\rho^{*} / n+b^{*} / s\right)+M^{*}\left(1-2 \alpha \rho^{*}\right)\right] \omega^{2} \\
\times & \left(\left(\lambda^{*}+2\right)\left(1-\left(\omega^{2} / s\right) \eta\right) M^{*}\right)^{-1}, \\
\alpha_{2}= & \frac{\left[\left(\rho^{*} / n+b^{*} / s\right)-\left(\rho^{*}\right)^{2}\right] \omega^{4}}{\left(\lambda^{*}+2\right)\left(1-\left(\omega^{2} / s\right) \eta\right) M^{*}} .
\end{aligned}
$$

The general solutions of $U(r) s /\left(s^{2}+\omega^{2}\right)$ and $W(r) s /\left(s^{2}+\right.$ $\left.\omega^{2}\right)$ in (25) are

$$
\begin{gathered}
\frac{U(r) s}{s^{2}+\omega^{2}}=A_{1} e^{-\gamma_{1} r}+A_{2} e^{-\gamma_{2} r}+A_{3} e^{\gamma_{1} r}+A_{4} e^{\gamma_{2} r}, \\
\frac{W(r) s}{s^{2}+\omega^{2}}=B_{1} e^{-\gamma_{1} r}+B_{2} e^{-\gamma_{2} r}+B_{3} e^{\gamma_{1} r}+B_{4} e^{\gamma_{2} r} .
\end{gathered}
$$

Considering the limitation property of radial displacement when $r \rightarrow \infty$, that is,

$U(r) \longrightarrow \infty \quad$ as $r \longrightarrow \infty, \quad W(r) \longrightarrow \infty \quad$ as $r \longrightarrow \infty$.

In (28), we have, $A_{3}=A_{4}=B_{3}=B_{4}$,

$$
\begin{aligned}
& \frac{U(r) s}{s^{2}+\omega^{2}}=A_{1} e^{-\gamma_{1} r}+A_{2} e^{-\gamma_{2} r}, \\
& \frac{W(r) s}{s^{2}+\omega^{2}}=B_{1} e^{-\gamma_{1} r}+B_{2} e^{-\gamma_{2} r} .
\end{aligned}
$$


Constants $A_{1}, A_{2}, B_{1}$, and $B_{2}$ in (30) and (31) are linearly dependent and may be related by using (25) to obtain

$$
B_{i}=\delta_{i} A_{i}, \quad i=1,2,
$$

where $\delta_{i}=-\left(\alpha M \gamma_{i}^{2}+\rho^{*} \omega^{2}\right) /\left(M^{*} \gamma_{i}^{2}+\left(\rho^{*} / n+b^{*} / s\right) \omega^{2}\right)$.

$A_{1}, A_{2}$ can be obtained from boundary conditions. Now,

$$
u_{r}=\frac{\partial}{\partial r}\left(\frac{U(r) \cos \omega t}{r}\right)
$$

The Laplace transformed solution of radial displacement $u_{r}$, that is, $\bar{u}_{r}$ is given by

$$
\begin{aligned}
\bar{u}_{r} & =\frac{\partial}{\partial r}\left(\frac{U(r) s}{r\left(s^{2}+\omega^{2}\right)}\right) \\
& =\frac{\partial}{\partial r} \frac{1}{r}\left(A_{1} e^{-\gamma_{1} r}+A_{2} e^{-\gamma_{2} r}\right), \quad \text { by }(30) \\
& =-\frac{A_{1}}{r}\left(\gamma_{1}+\frac{1}{r}\right) e^{-\gamma_{1} r}-\frac{A_{2}}{r}\left(\gamma_{2}+\frac{1}{r}\right) e^{-\gamma_{2} r}
\end{aligned}
$$

Similarly, the Laplace transform solution of $w_{r}$ is $\bar{w}_{r}$ :

$$
\begin{aligned}
w_{r}= & \frac{\partial}{\partial r}\left(\frac{W(r) \cos \omega t}{r}\right), \\
\bar{w}_{r}= & \frac{\partial}{\partial r}\left(\frac{W(r) s}{r\left(s^{2}+\omega^{2}\right)}\right) \\
= & \frac{\partial}{\partial r} \frac{1}{r}\left(B_{1} e^{-\gamma_{1} r}+B_{2} e^{-\gamma_{2} r}\right), \\
= & -\frac{1}{r}\left(\gamma_{1}+\frac{1}{r}\right) e^{-\gamma_{1} r} B_{1}-\frac{1}{r}\left(\gamma_{2}+\frac{1}{r}\right) e^{-\gamma_{2} r} B_{2} \\
= & -\frac{\delta_{1}}{r}\left(\gamma_{1}+\frac{1}{r}\right) e^{-\gamma_{1} r} A_{1} \\
& -\frac{\delta_{2}}{r}\left(\gamma_{2}+\frac{1}{r}\right) e^{-\gamma_{2} r} A_{2}, \quad \text { by (32). }
\end{aligned}
$$

Next, by (5),

$$
\begin{aligned}
p & =M \xi-\alpha M e \\
& =-M\left(\frac{\partial w_{r}}{\partial r}+\frac{2 w_{r}}{r}\right)-\alpha M\left(\frac{\partial u_{r}}{\partial r}+\frac{2 u_{r}}{r}\right) \\
\frac{P}{G} & =-M^{*}\left(\frac{\partial w_{r}}{\partial r}+\frac{2 w_{r}}{r}\right)-\alpha M^{*}\left(\frac{\partial u_{r}}{\partial r}+\frac{2 u_{r}}{r}\right) .
\end{aligned}
$$

Taking Laplace transform and using (34) and (35), we have

$$
\begin{aligned}
& \left(\frac{\bar{P}}{\bar{G}}\right)=-M^{*}\left[\frac{\partial}{\partial r}\left\{-\sum_{i=1}^{2} \frac{\delta_{i}}{r}\left(\gamma_{i}+\frac{1}{r}\right) e^{-\gamma_{i} r} A_{i}\right\}\right. \\
& \left.+\frac{2}{r}\left\{-\sum_{i=1}^{2} \frac{\delta_{i}}{r}\left(\gamma_{i}+\frac{1}{r}\right) e^{-\gamma_{i} r} A_{i}\right\}\right] \\
& -\alpha M^{*}\left[\frac{\partial}{\partial r}\left\{-\sum_{i=1}^{2} \frac{1}{r}\left(\gamma_{i}+\frac{1}{r}\right) e^{-\gamma_{i} r} A_{i}\right\}\right. \\
& \left.+\frac{2}{r}\left\{-\sum_{i=1}^{2} \frac{1}{r}\left(\gamma_{i}+\frac{1}{r}\right) e^{-\gamma_{i} r} A_{i}\right\}\right] \\
& =\sum_{i=1}^{2}\left(\alpha+\delta_{i}\right) M^{*} \\
& \times A_{i}\left[-\frac{1}{r^{2}}\left(\gamma_{i}+\frac{1}{r}\right) e^{-\gamma_{i} r}-\frac{1}{r^{3}} e^{-\gamma_{i} r}\right. \\
& +\frac{2}{r}\left(\gamma_{i}+\frac{1}{r}\right) e^{-\gamma_{i} r}\left(-\gamma_{i}\right) \\
& \left.+\frac{2}{r}\left(\gamma_{i}+\frac{1}{r}\right) e^{-\gamma_{i} r}\right], \\
& \left(\frac{\bar{P}}{\bar{G}}\right)=-\sum_{i=1}^{2}\left(\alpha+\delta_{i}\right) \frac{1}{r} M^{*} \gamma_{i}^{2} e^{-\gamma_{i} r} A_{i}
\end{aligned}
$$

Next, by (3),

$$
\begin{aligned}
\frac{\sigma_{r}}{G}= & \lambda^{*}\left(\frac{\partial u_{r}}{\partial r}+\frac{2 u_{r}}{r}\right)+2 \frac{\partial u_{r}}{\partial r} \\
& +\lambda^{*} \eta \frac{\partial}{\partial t}\left(\frac{\partial u_{r}}{\partial r}+\frac{2 u_{r}}{r}\right)+2 \eta \frac{\partial}{\partial t}\left(\frac{u_{r}}{r}\right)-\alpha \frac{P}{G}
\end{aligned}
$$

Taking Laplace transform of both sides

$$
\begin{aligned}
\left(\frac{\bar{\sigma}_{r}}{G}\right)= & \lambda^{*}\left(\frac{\partial \bar{u}_{r}}{\partial r}+\frac{2 \bar{u}_{r}}{r}\right)+2 \frac{\partial \bar{u}_{r}}{\partial r} \\
& +\lambda^{*} \eta\left(\frac{\partial}{\partial r}\left(\frac{-\omega^{2}}{s} \bar{u}_{r}\right)-\frac{2 \omega^{2}}{s} \frac{\bar{u}_{r}}{r}\right) \\
& +2 \eta \frac{\partial}{\partial r}\left(\frac{-\omega^{2}}{s} \bar{u}_{r}\right)-\alpha \frac{\bar{P}}{G} \\
= & \left(\lambda^{*}+2\right)\left(1-\frac{\omega^{2}}{s} \eta\right) \frac{\partial \bar{u}_{r}}{\partial r} \\
& +2 \lambda^{*}\left(1-\frac{\omega^{2}}{s} \eta\right) \frac{\bar{u}_{r}}{r}-\alpha \frac{\bar{P}}{G}
\end{aligned}
$$


6

ISRN Mechanical Engineering

$$
\begin{aligned}
& =\sum_{i=1}^{2}\left(\lambda^{*}+2\right)\left(1-\frac{\omega^{2}}{s} \eta\right) \frac{\partial}{\partial r}\left(-\frac{1}{r}\left(\gamma_{i}+\frac{1}{r}\right) e^{-\gamma_{i} r}\right) \\
& +\frac{2 \lambda^{*}}{r}\left(1-\frac{\omega^{2}}{s} \eta\right) \sum_{i=1}^{2}\left(-\frac{1}{r}\left(\gamma_{i}+\frac{1}{r}\right) e^{-\gamma_{i} r} A_{i}\right) \\
& +\alpha \sum_{i=1}^{2}\left(\alpha+\delta_{i}\right) A_{i} M^{*} \gamma_{i}^{2} e^{-\gamma_{i} r}, \quad \text { by (34) and (35) } \\
& =-\sum_{i=1}^{2}\left(\lambda^{*}-2\right)\left(1-\frac{\omega^{2}}{s} \eta\right) \\
& \times A_{i}\left\{-\frac{1}{r^{2}}\left(\gamma_{i}+\frac{1}{r}\right) e^{-\gamma_{i} r}-\frac{1}{r^{3}} e^{-\gamma_{i} r}\right. \\
& \left.+\frac{1}{r}\left(\gamma_{i}+\frac{1}{r}\right) e^{-\gamma_{i} r}\left(-\gamma_{i}\right)\right\} \\
& -2 \lambda^{*} \sum_{i=1}^{2}\left(1-\frac{\omega^{2}}{s} \eta\right) \frac{1}{r^{2}}\left(\gamma_{i}+\frac{1}{r}\right) e^{-\gamma_{i} r} A_{i} \\
& +\alpha \sum_{i=1}^{2} \frac{1}{r}\left(\alpha+\delta_{i}\right) A_{i} M^{*} \gamma_{i}^{2} e^{-\gamma_{i} r} \\
& =\sum_{i=1}^{2}\left(\lambda^{*}+2\right)\left(1-\frac{\omega^{2}}{s} \eta\right) e^{-\gamma_{i} r} A_{i} \gamma_{i}^{2} \\
& +2 \sum_{i=1}^{2}\left(\lambda^{*}+2\right)\left(1-\frac{\omega^{2}}{s} \eta\right) e^{-\gamma_{i} r} \frac{A_{i}}{r^{2}}\left(\gamma_{i}+\frac{1}{r}\right) \\
& -2 \sum_{i=1}^{2} \lambda^{*}\left(1-\frac{\omega^{2}}{s} \eta\right)+e^{-\gamma_{i} r} \frac{A_{i}}{r^{2}}\left(\gamma_{i}+\frac{1}{r}\right) \\
& +\alpha \sum_{i=1}^{2} \frac{1}{r}\left(\alpha+\delta_{i}\right) A_{i} M^{*} \gamma_{i}^{2} e^{-\gamma_{i} r} \\
& \left(\frac{\bar{\sigma}_{r}}{G}\right)=A_{i} \sum_{i=1}^{2}\left[\frac{1}{r}\left(\lambda^{*}+2\right)\left(1-\frac{\omega^{2}}{s} \eta\right) \gamma_{i}^{2} \frac{4}{r^{2}}\left(1-\frac{\omega^{2}}{s} \eta\right)\right. \\
& \left.\times\left(\gamma_{i}+\frac{1}{r}\right)+\frac{\alpha}{r} \gamma_{i}^{2}\left(\alpha+\delta_{i}\right) M^{*}\right] .
\end{aligned}
$$

Similarly, by (4), (35), and (37), we have

$$
\begin{aligned}
\left(\frac{\sigma_{\theta}}{G}\right)= & \left(\frac{\sigma_{\phi}}{G}\right) \\
= & \lambda^{*} e+2 \frac{u_{r}}{r}+\eta \lambda^{*} \frac{\partial e}{\partial t}+2 \eta \frac{\partial}{\partial t}\left(\frac{u_{r}}{r}\right)-\alpha \frac{p}{G} \\
= & \lambda^{*}\left(\frac{\partial u_{r}}{\partial r}+\frac{2 u_{r}}{r}\right)+\frac{2 u_{r}}{r}+\eta \lambda^{*} \frac{\partial}{\partial t}\left(\frac{\partial u_{r}}{\partial r}+\frac{2 u_{r}}{r}\right) \\
& +2 \eta \frac{\partial}{\partial t}\left(\frac{u_{r}}{r}\right)-\alpha \frac{p}{G} .
\end{aligned}
$$

Then

$$
\begin{aligned}
L\left(\frac{\sigma_{\theta}}{G}\right) & \left(\frac{\bar{\sigma}_{\theta}}{G}\right) \\
= & \lambda^{*}\left(\frac{\partial \bar{u}_{r}}{\partial r}+\frac{2 \bar{u}_{r}}{r}\right)+\frac{2 \bar{u}_{r}}{r} \\
& +\eta \lambda^{*}\left(-\frac{\omega^{2}}{s} \frac{\partial \bar{u}_{r}}{\partial r}-\frac{2 \omega^{2}}{s} \frac{\bar{u}_{r}}{r}\right) \\
& +2 \eta\left(-\frac{\omega^{2}}{s} \frac{\bar{u}_{r}}{r}\right)-\alpha \frac{\bar{p}}{G} \\
= & \lambda^{*}\left(1-\frac{\omega^{2}}{s} \eta\right) \frac{\partial \bar{u}_{r}}{\partial r} \\
& +2\left(\lambda^{*}+1\right)\left(1-\frac{\omega^{2}}{s} \eta\right) \frac{\bar{u}_{r}}{r}-\alpha\left(\frac{\bar{p}}{G}\right) \\
= & -\lambda^{*}\left(1-\frac{\omega^{2}}{s} \eta\right) \sum_{i=1}^{2} \frac{\partial}{\partial r} \frac{1}{r}\left(\gamma_{i}+\frac{1}{r}\right) e^{-\gamma_{i} r} A_{i} \\
& -2\left(\lambda^{*}+1\right)\left(1-\frac{\omega^{2}}{s} \eta\right) \\
& \times \sum_{i=1}^{2} \frac{1}{r^{2}}\left(\gamma_{i}+\frac{1}{r}\right) e^{-\gamma_{i} r} A_{i}-\alpha\left(\frac{\bar{p}}{G}\right) \\
= & -\lambda^{*}\left(1-\frac{\omega^{2}}{s} \eta\right) \\
& \times \sum_{i=1}^{2}\left[-\frac{1}{r^{2}}\left(\gamma_{i}+\frac{1}{r}\right)-\frac{1}{r^{3}}-\frac{\gamma_{i}}{r}\left(\gamma_{i}+\frac{1}{r}\right)\right] A_{i} e^{-\gamma_{i} r} \\
& -2\left(\lambda^{*}+1\right)\left(1-\frac{\omega^{2}}{s} \eta\right) \\
= &
\end{aligned}
$$

$$
\begin{aligned}
\times \sum_{i=1}^{2} \frac{1}{r^{2}}\left(\gamma_{i}+\frac{1}{r}\right) e^{-\gamma_{i} r} A_{i}-\alpha\left(\frac{\bar{p}}{G}\right) \\
=\sum_{i=1}^{2} \frac{\lambda^{*}}{r}\left(1-\frac{\omega^{2}}{s} \eta\right) \gamma_{i}^{2} e^{-\gamma_{i} r} A_{i} \\
-\sum_{i=1}^{2}\left(1-\frac{\omega^{2}}{s} \eta\right) \\
\times\left[-\frac{2 \lambda^{*}}{r^{2}}\left(\gamma_{i}+\frac{1}{r}\right)+\frac{2 \lambda^{*}}{r^{2}}\left(\gamma_{i}+\frac{1}{r}\right)+\frac{2}{r^{2}}\right]
\end{aligned}
$$

(40)

$-\alpha\left(\frac{\bar{p}}{G}\right)$ 


$$
\begin{gathered}
=\sum_{i=1}^{2}\left[\frac{\lambda^{*}}{r}\left(1-\frac{\omega^{2}}{s} \eta\right) \gamma_{i}^{2}-\frac{2}{r^{2}}\left(1-\frac{\omega^{2}}{s} \eta\right)\left(\gamma_{i}+\frac{1}{r}\right)\right. \\
\left.+\frac{\alpha}{r} \gamma_{i}^{2}\left(\alpha+\delta_{i}\right) M^{*}\right] e^{-\gamma_{i} r} A_{i} .
\end{gathered}
$$

\section{Solution of Shell Embedment and Axisymmetric Loading}

Dynamic loads applied on the surface of shell considered herein are an axially symmetric radial traction and axially symmetric fluid pressure with the step style shown in Figure 2, where $T^{*}$ is the nondimensional step load time $\left(T^{*}=T \sqrt{G / \rho} / b\right), T$ is actual step load time; $\left(t^{*}=t \sqrt{G / \rho} / b\right)$, the nondimensional time; $t$ is actual time; $q_{0}$ is maximum of the step load. In the domain of Laplace transform, the load can be expressed as

$$
\bar{q}(s)=\frac{q_{0}}{T^{*}}\left(\frac{1-e^{-T^{*} s}}{s^{2}}\right), \quad r=b .
$$

Here, the case of a thin, elastic shell embedded in infinite viscoelastic saturated soil subjected to axisymmetric surface load and fluid pressure is considered. The equation of motion of this shell under nontorsional axisymmetric loading is

$$
2\left(1+\mu_{1}\right) \mu_{r}^{\prime}+\gamma_{0}^{2} \frac{\partial^{2} \mu_{r}^{\prime}}{\partial t^{2}}=q_{0}(t) \frac{\left(a^{\prime}\right)\left(1-\mu_{1}^{2}\right)}{E_{1} h},
$$

where $\gamma_{0}^{2}=c_{1}^{2} / c_{p}^{2} ; c_{1}=\sqrt{(\lambda+2 G) / \rho}$ and $c_{p}=$ $\sqrt{E_{1} /\left(\rho_{f}\left(1-\mu^{2}\right)\right)}$ are the dilatational wave velocity and plate velocity, respectively; $E_{1}, \mu_{1}$ are the modulus and Poission's rate of shell, respectively; $q_{0}(t)$ is the net outward radial pressure. For a thin shell, the thick $h / 2$ can be omitted without significant error. The interface shell and soil can be defined as $r=a+h / 2=a^{\prime}$. The stress and displacement condition at the interface is expressed as

$$
\begin{gathered}
q_{0}(t)=q(t)-\sigma_{r}, \quad r=a+\frac{h}{2}=a^{\prime}, \\
u_{r}=-u_{r}^{\prime}, \quad r=a+\frac{h}{2}=a^{\prime},
\end{gathered}
$$

where $q(t)$ is the radial stress applied at the inner surface of the shall; $\sigma_{r}$ is the stress exerted by the soil on the shell and can be given by (39).

In practical situation, the condition is frequently found in two extreme cases; permeable and impermeable.

The partial permeable flow boundary condition is

$$
\frac{\partial p}{\partial r}=\frac{k p}{a^{\prime}} \quad \text { at } r=a+\frac{h}{2}=a^{\prime},
$$

where $k=\left(k_{1} / k_{d}\right)(1 / \log (b / a))$ is a dimensionless permeability parameter that defines the flow capacity of the shell. The parameter $k$ depends on the relative permeability of the shell and soil as well as the geometry of the shell, that is,
(1) when the spherical shell is impermeable, that is, $k_{1}=$ $0, k$ tends to zero and

(2) when the shell is permeable, that is, $k_{1}=\infty, k$ tends to infinity.

Substituting (34), (39), (44) into (43) and (37) into (46), we obtain

$$
\begin{gathered}
m_{1} A_{1}+m_{2} A_{2}=\frac{\left(a^{\prime}\right)^{2}\left(1-\mu_{1}^{2}\right)}{E_{1}^{*} h} \frac{\bar{q}(s)}{G} \\
n_{1} A_{1}+n_{2} A_{2}=0
\end{gathered}
$$

where

$$
\begin{aligned}
m_{i}= & {\left[2\left(1+\mu_{1}\right)+\gamma_{0}^{2} \omega^{2}\right] \frac{1}{a^{\prime}}\left(\gamma_{i}+\frac{1}{a^{\prime}}\right) e^{-\gamma_{i} a^{\prime}} } \\
+ & {\left[\frac{1}{a^{\prime}}\left(\lambda^{*}+2\right)\left(1-\frac{\omega^{2}}{s} \eta\right)\right.} \\
& \times \gamma_{i}^{2} \frac{4}{\left(a^{\prime}\right)^{2}}\left(1-\frac{\omega^{2}}{s} \eta\right)\left(\gamma_{i}+\frac{1}{a^{\prime}}\right) \\
& \left.+\frac{\alpha}{a^{\prime}} \gamma_{i}^{2}\left(\alpha+\delta_{i}\right) M^{*}\right] \frac{\left(a^{\prime}\right)^{2}\left(1-\mu_{1}^{2}\right)}{E_{1}^{*} h} e^{-\gamma_{i} a^{\prime}} \\
n_{i}= & \frac{1}{a^{\prime}}\left(\gamma_{i}+\frac{k+1}{a^{\prime}}\right)\left(\alpha+\delta_{i}\right) M^{*} \gamma_{i}^{2} e^{-\gamma_{i} a^{\prime}}, \quad i=1,2, \\
E_{i}^{*}= & \frac{E_{1}}{G}, \\
A_{1}= & -\frac{n_{2}}{n_{1}} A_{2}, \\
A_{2}= & -\left(\frac{n_{1}}{n_{1} m_{2}-n_{2} m_{1}}\right) \frac{\left(a^{\prime}\right)^{2}\left(1-\mu_{1}^{2}\right)}{E_{1}^{*} h} \frac{\bar{q}(s)}{G} .
\end{aligned}
$$

Under the fluid pressure on shell surface, the displacement and stress components are continuous at the kinematic interface between the spherical shell and soil. In this case, flow boundary conditions are

$$
\begin{gathered}
q_{0}(t)=-\sigma_{r} \quad \text { at } r=a+\frac{h}{2}=a^{\prime}, \\
u_{r}=-u_{r}^{\prime} \quad \text { at } r=a+\frac{h}{2}=a^{\prime}, \\
\frac{\partial p}{\partial r}=\frac{k}{a}(p+q(t)) \quad \text { at } r=a+\frac{h}{2}=a^{\prime} .
\end{gathered}
$$

Substituting (34), (39), (49), (50) into (43) and (37) into (51) yields

$$
\begin{gathered}
m_{1} A_{1}+m_{2} A_{2}=0, \\
n_{1} A_{1}+n_{2} A_{2}=\frac{k}{a^{\prime}} \frac{\bar{q}(s)}{G},
\end{gathered}
$$


TABLE 1: Parameters used in computation.

\begin{tabular}{lcc}
\hline Quantity & Notation & Value \\
\hline Elastic modulus of soil (MPa) & $E_{s}$ & 7 \\
Poisson rate of soil & $\mu$ & 0.35 \\
Poisson rate of shell & $\mu_{l}$ & 0.09 \\
Dimensionless shell density & $\rho_{l}^{*}$ & 1.4 \\
Dimensionless shell thickness & $h / a$ & 0.04 \\
Dimensionless fluid density & $\rho^{*}$ & 0.45 \\
Compressible parameter of material & $\alpha$ & 0.96 \\
Dimensionless compressible parameter & $M^{*}$ & 18 \\
Dimensionless permeability coefficient & $b^{*}$ & 9 \\
Viscoelastic damp coefficient & $\eta$ & 0.35 \\
Porosity & $n$ & 0.35 \\
Gradually applied step load time & $T^{*}$ & 1 \\
\hline
\end{tabular}

or

$$
\begin{gathered}
A_{1}=-\frac{m_{1}}{m_{2}} A_{2}, \\
A_{2}=\frac{k}{a^{\prime}}\left(\frac{m_{1}}{m_{1} n_{2}-m_{2} n_{1}}\right) \frac{\bar{q}(s)}{G} .
\end{gathered}
$$

Using inverse Laplace transform and numerical computation, the final solutionin time domain can be obtained after determining $A_{1}$ and $A_{2}$.

\section{Results and Discussion}

In this paper, we will discuss the influences of partial permeable property of boundary and relative rigidity of shell and soil (defined as $\mathrm{RR}=E_{l} / E_{s}$ ) on the transient response of the spherical cavity. The numerical results are presented for the material and geometric parameters which are listed in Table 1 .

4.1. Solutions Corresponding to Fluid Pressure. The histories of dimensionless radial displacement under fluid pressure are shown in Figure 2 when parameters $k=0.5$. It is noted that at a certain time instant as shown in Figure 2, there exists maximum displacement at the interface of shell and soil. With the increase of time, radial displacement decreased vibrationally and finally to an asymptotic value of zero. Radial displacement decreased obviously with increasing relative rigidity, and increased with increasing of parameter $k$ (Figure 3).

The excess pore pressures induced by fluid pressure are shown in Figure 4. However, the influence of parameter $k$ (Figure 4) is significant. When the shell boundary became almost impermeable $(k \rightarrow 0)$, almost no excess pore pressure existed, whereas with the increasing of time, the excess pore pressure at the interface equaled the fluid pressure $(P=$ $-q_{0}$ ) when the shell boundary became almost permeable $(k \rightarrow \infty)$.

4.2. Solutions Corresponding to Radial Load. The histories of dimensionless radial displacement at the interface of shell

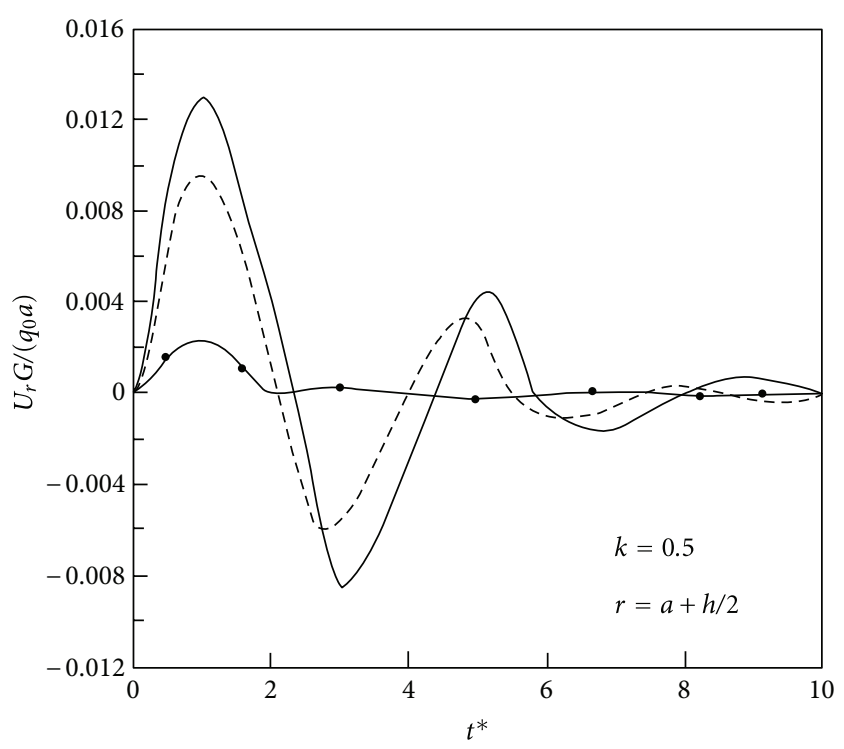

$$
\begin{aligned}
\longrightarrow R R & =5 \\
--R R & =20 \\
\multimap & R R=100
\end{aligned}
$$

FIGURE 2: Curve of radial displacement versus relative rigidity (fluid pressure).

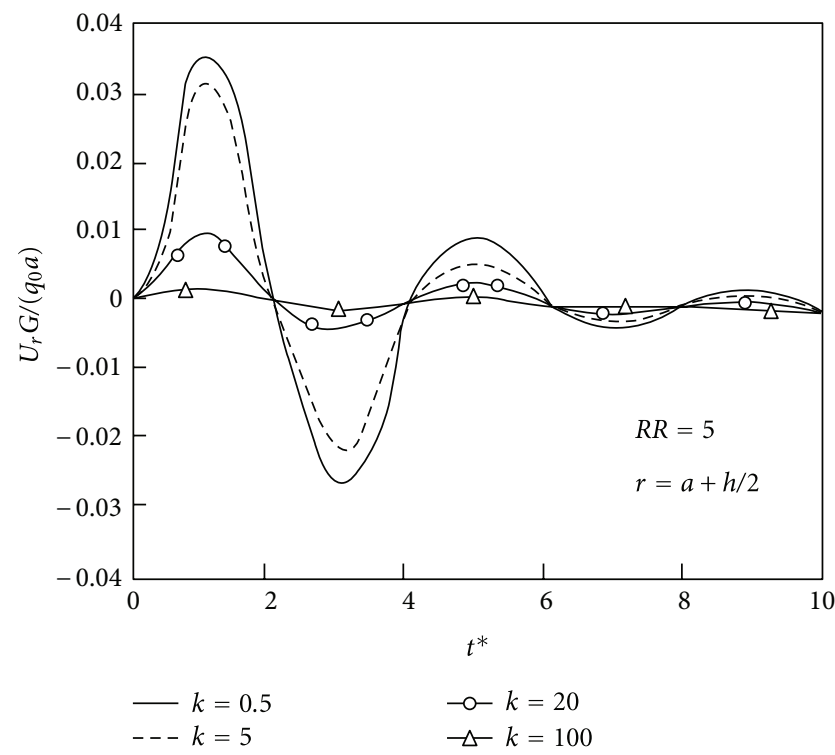

FIGURE 3: Curve of radial displacement versus parameter $k$ (fluid pressure).

and soil induced by axially symmetric radial surface load are shown in Figure 5 when the parameter $k=0.5$. With the increase of the dimensionless time $\left(t^{*}=t \sqrt{G / \rho / a}\right)$, radial displacement increases to maximum value, then decreases and is noted once again. Eventually, it tends to an asymptotic value. When relative rigidity $R R=0$, the shell is complete flexible, there is the maximum radial displacement at the interface of shell and soil. The value of radial displacement 


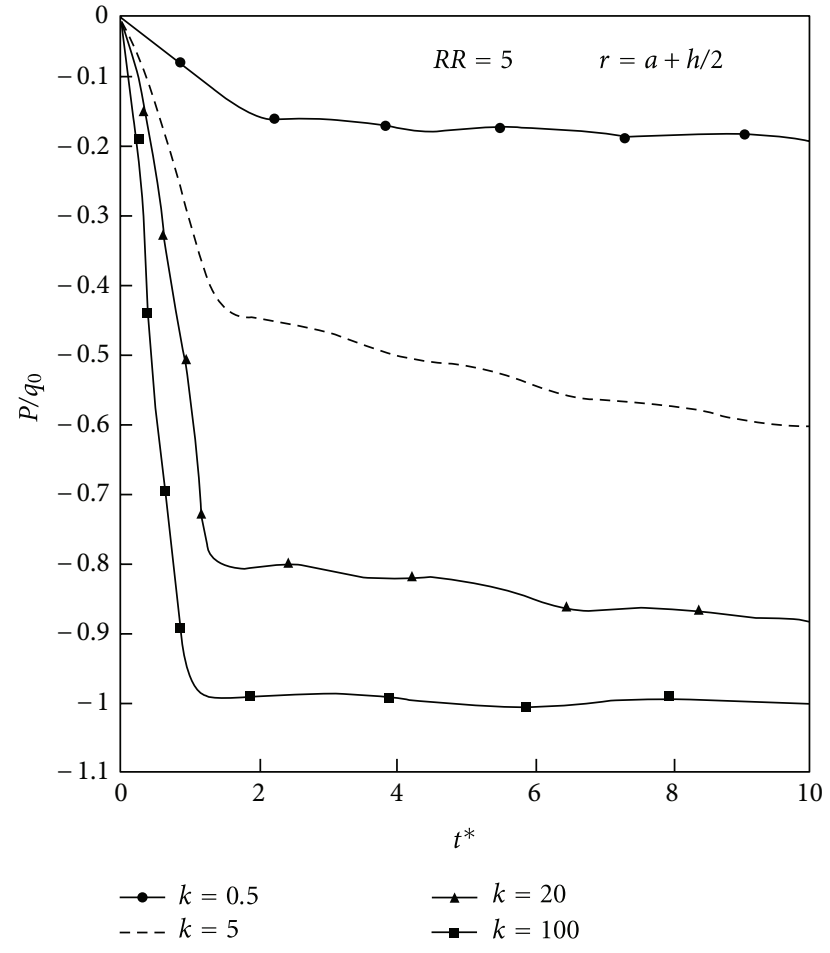

Figure 4: Curve of pore pressure versus parameter $k$ (fluid pressure).

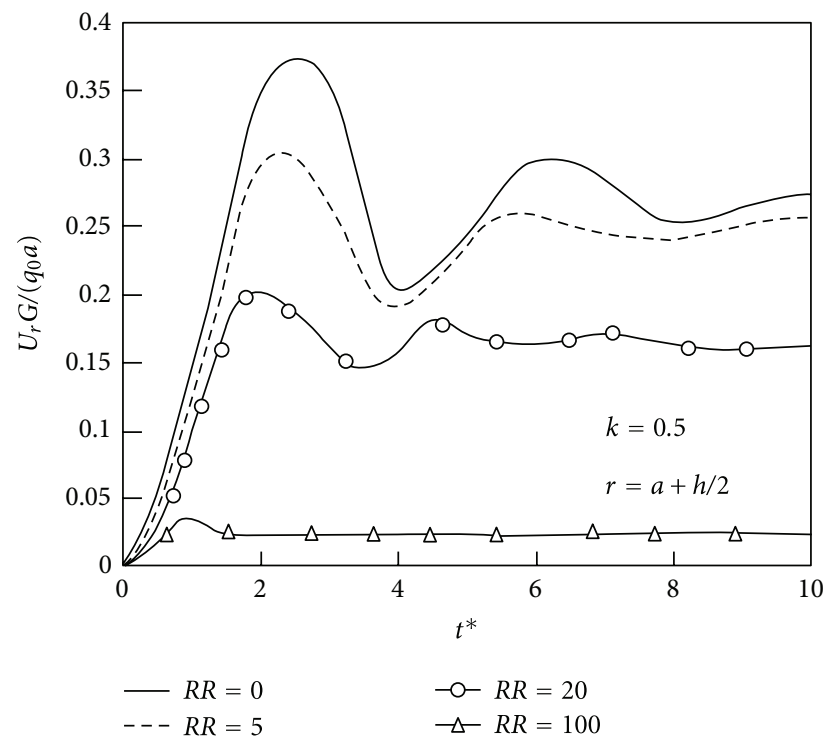

FIgURE 5: Curve of radial displacement versus relative rigidity.

decreases with the increase of relative rigidity. The influence of permeability parameter $k$ on radial displacement is indicated in Figure 6. It can be seen that the influence of parameter $k$ on radial displacement induced by axisymmetric radial surface load is not remarkable.

The histories of dimensionless pore pressure are shown in Figure 7 at the interface of shell and soil for the parameter $k=0.5$. Pore pressure is zero at $t^{*}=0$ and increases rapidly

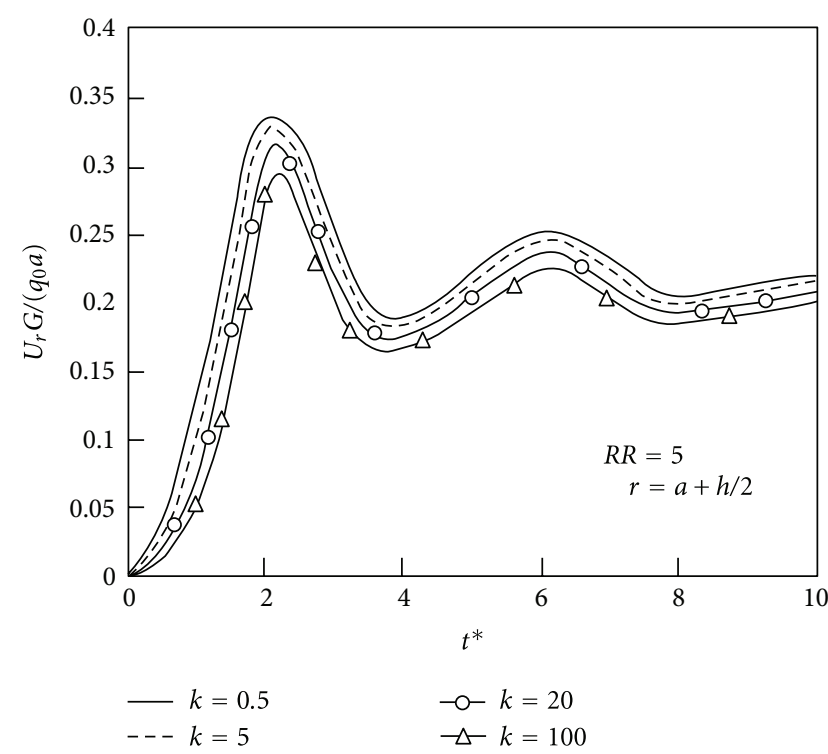

FIGURE 6: Curve of radial displacement versus parameter $k$.

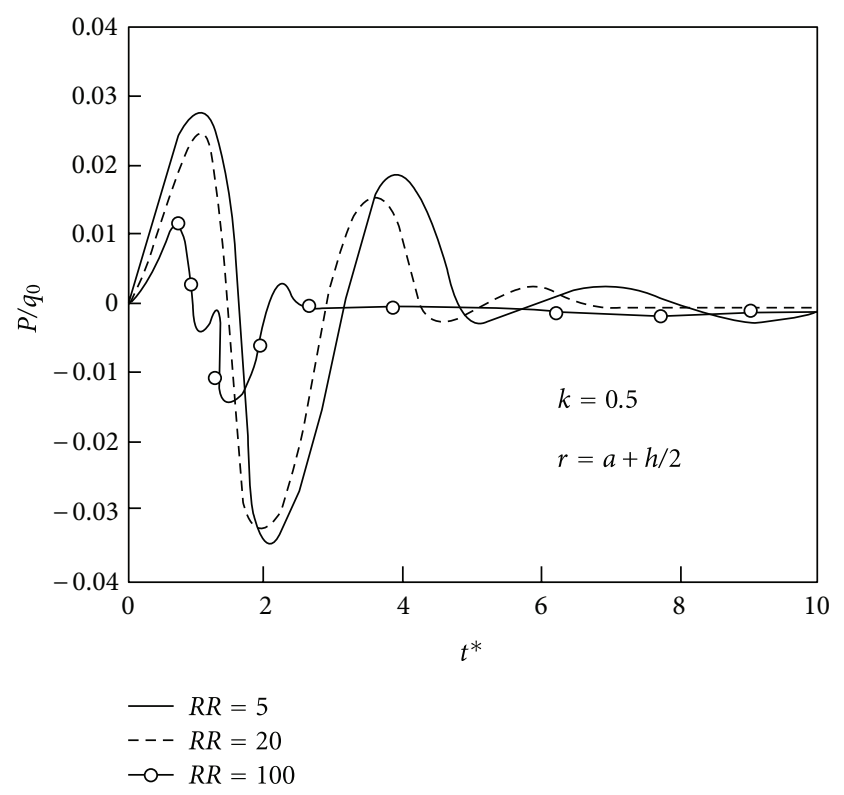

FIGURE 7: Curve of pore pressure versus relative rigidity.

with time in the interval $0<t^{*} \leq T^{*}$ and reaches to its peak value nearly at $t^{*}=T^{*}$. Thereafter, it decreases with time and reaches to its maximum suction values. With increasing time the values of suction decreases and pore pressure is noted once again. On the other hand, the pore pressure decreases with the increase of parameter $k$ (Figure 8). As a result, both the relative rigidity and parameter $k$ have great influence on the pore pressure under the condition of axisymmetric radial surface load. 


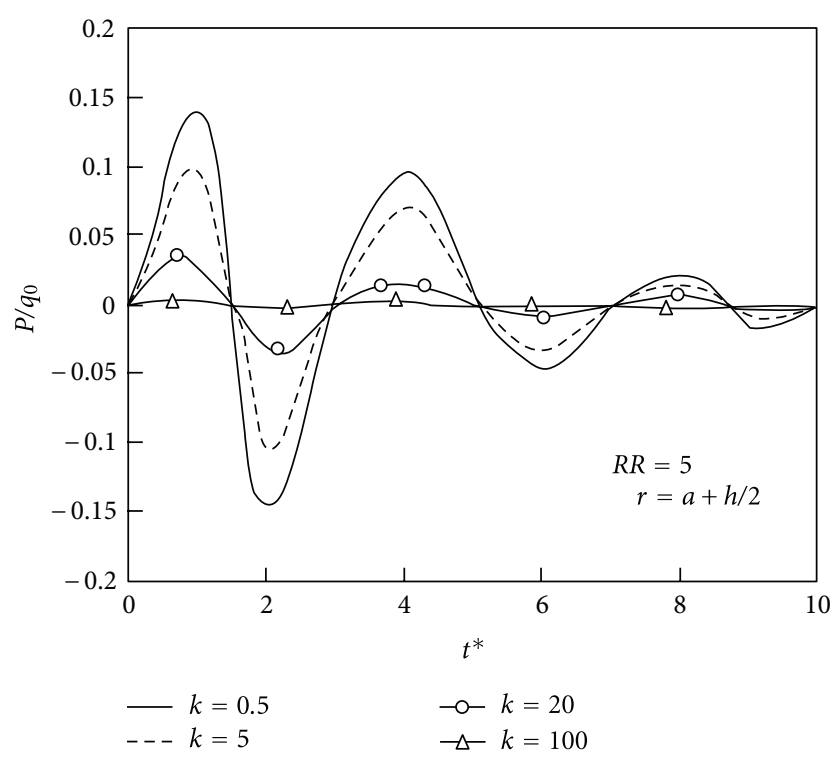

FIGURE 8: Curve of pore pressure versus parameter $k$.

\section{Conclusions}

An extensive parameters study conducted to investigate the influence of therelative rigidity of shell and soil and permeability parameter $k$, showed that permeability parameter $k$ depends on the relative permeability of the liner and soil as well as the geometry of the liner. Relative rigidity and parameter $k$ have significant influences on the transient response of spherical cavity with a shell embedded in viscoelastic saturated soil. The solutions under permeable and impermeable boundary conditions are only two extreme cases. Thus partially sealed boundary condition and the relative rigidity of shell and soil in the designing and computation of spherical shell in viscoelastic saturated medium are remarkable.

\section{References}

[1] M. A. Biot, "Theory of deformation of a porous viscoelastic anisotropic solid," Journal of Applied Physics, vol. 27, no. 5, pp. 459-467, 1956.

[2] M. A. Biot, "Mechanics of deformation and acoustic propagation in porous media," Journal of Applied Physics, vol. 33, no. 4, pp. 1482-1498, 1962.

[3] N. Akkas and U. Zakout, "Transient response of an infinite elastic medium containing a spherical cavity with and without a shell embedment," International Journal of Engineering Science, vol. 35, no. 2, pp. 89-112, 1997.

[4] A. C. Eringen, Mechanics of Continua, R. E. Krieger, New York, NY, USA, 1980.

[5] F. Durbin, "Numerical inversion of laplace transforms: an efficient improvement to dubner and abate's method," The Computer Journal, vol. 17, no. 4, pp. 371-376, 1974. 

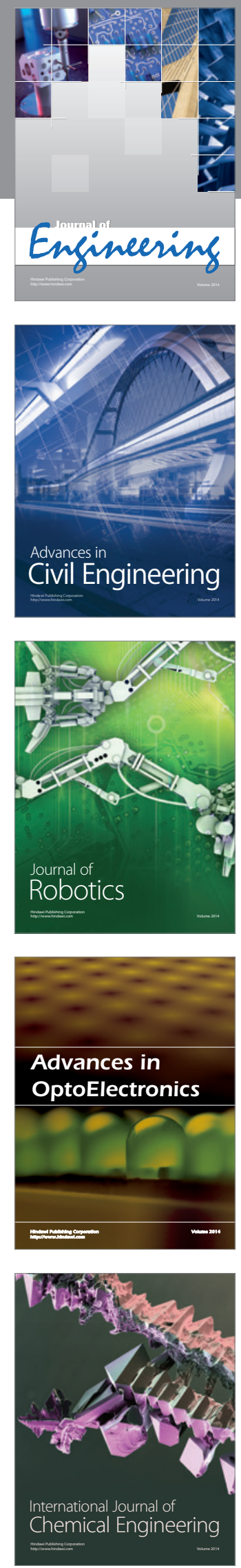

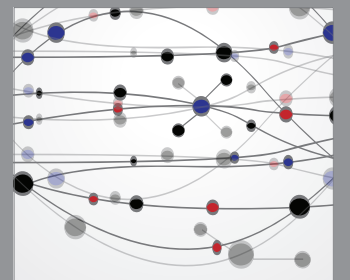

The Scientific World Journal
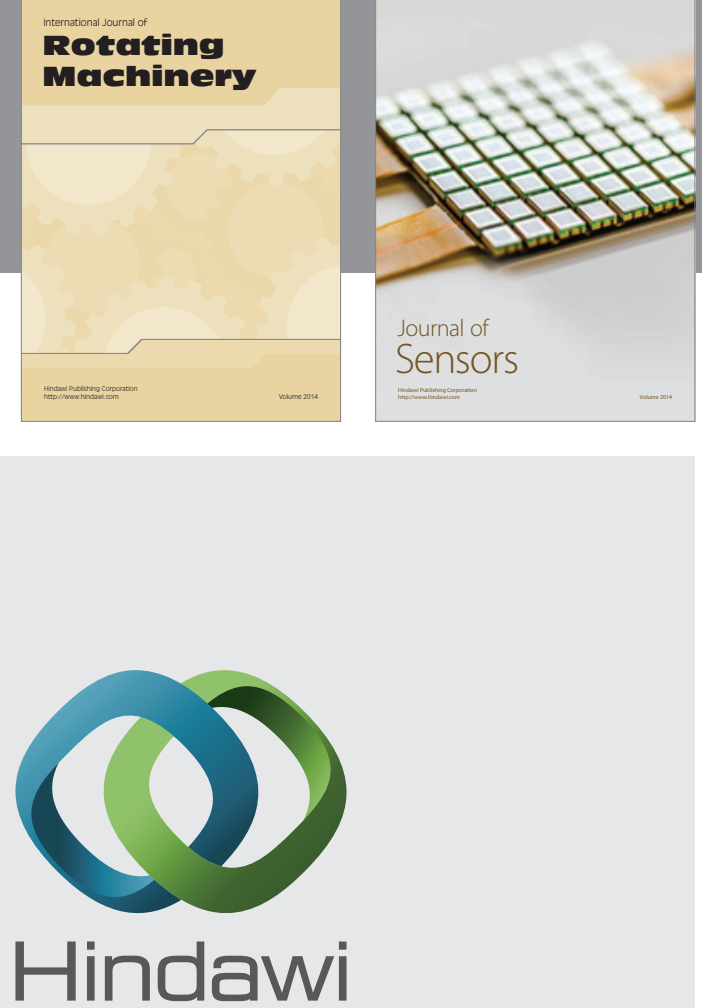

Submit your manuscripts at http://www.hindawi.com
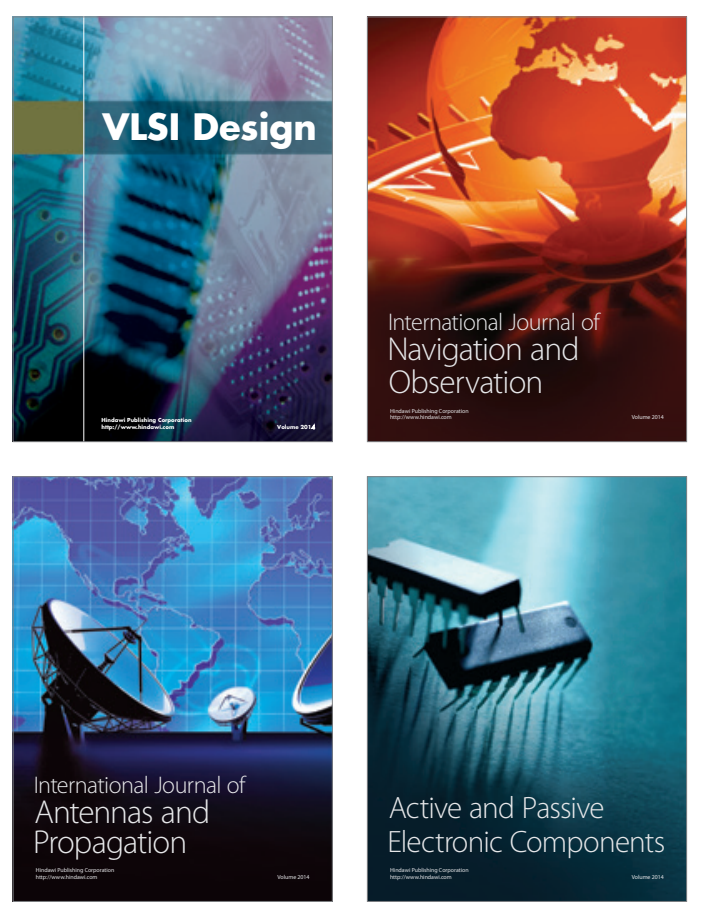
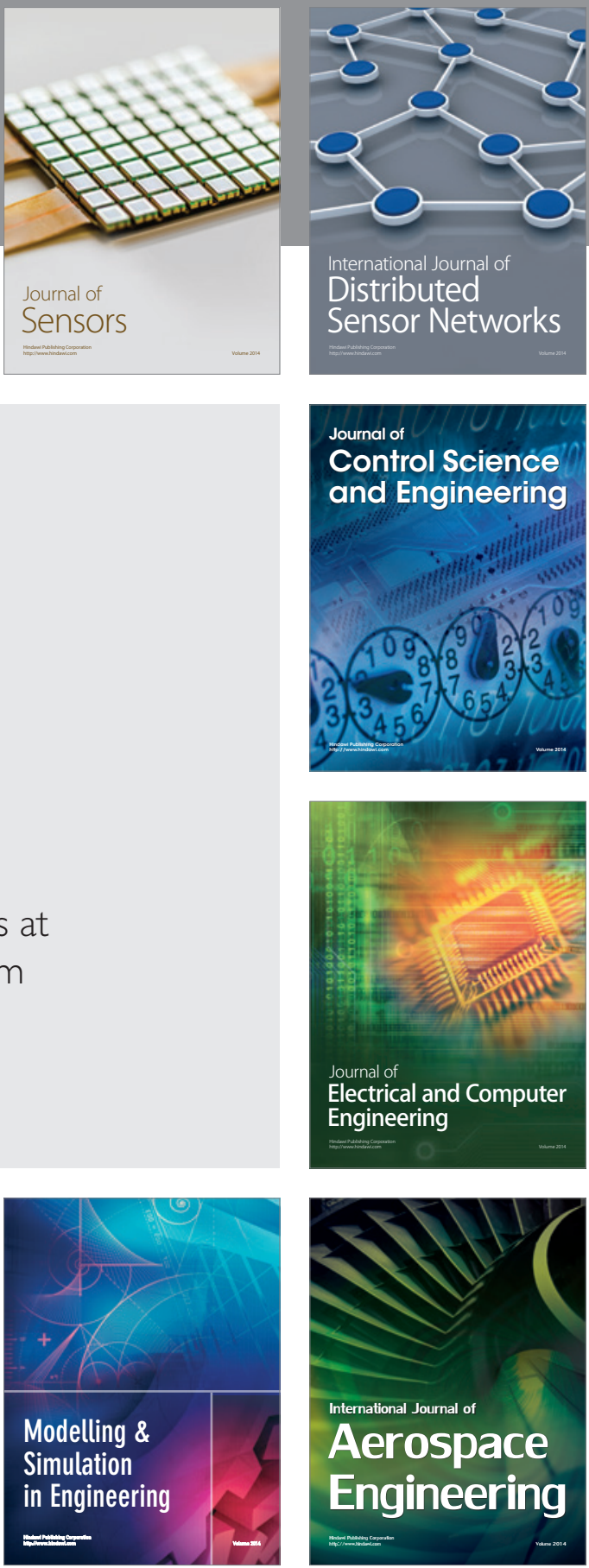

Journal of

Control Science

and Engineering
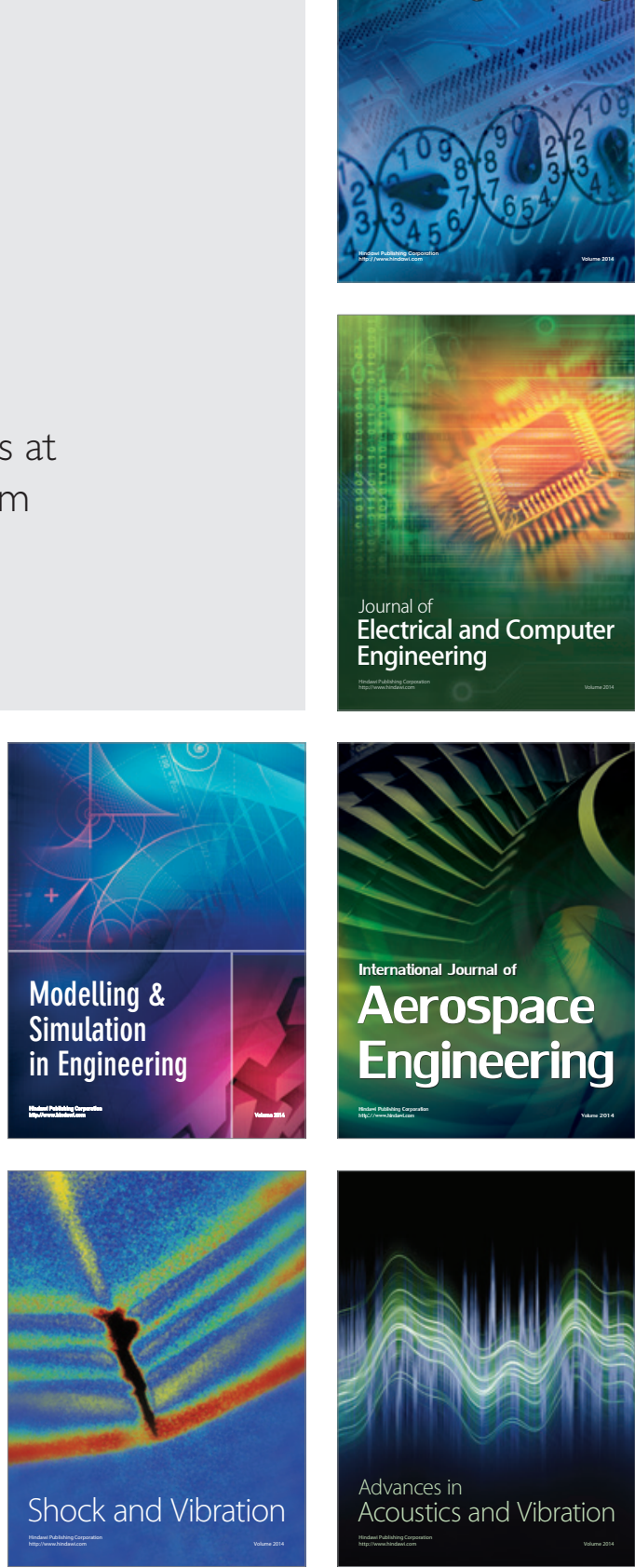